\title{
Practice of Advanced Medical Research of the Okayama University (National)
}

\author{
Kiyoshi MOROTA \\ Former President of Okayama University.
}

I would like to talk to you using the slides in the presentation. Firstly, I will introduce our university, and then I would like to explain to you the vision I proposed when I was inaugurated as the president of the university. I will then explain our motivation and objective for coming to this place at this time, and finally present our university as a global university proposal that we would like to make to you. 